\title{
What to Look Out for when Transplanting T-Cell Prolymphocytic Leukemia
}

\author{
Linus Wahnschaffe ${ }^{\mathrm{a}-\mathrm{c}}$ Marco Herling ${ }^{\mathrm{a}-\mathrm{c}}$ \\ ${ }^{a}$ Department I of Internal Medicine, Center for Integrated Oncology (CIO), Aachen-Bonn-Cologne-Duesseldorf, \\ University of Cologne, Cologne, Germany; ${ }^{\text {b }}$ Excellence Cluster for Cellular Stress Response and Aging-Associated \\ Diseases (CECAD), University of Cologne, Cologne, Germany; ${ }^{\circ}$ Center for Molecular Medicine Cologne (CMMC), \\ University of Cologne, Cologne, Germany
}

\section{Keywords}

T-cell prolymphocytic leukemia - Allogeneic stem cell transplantation - Late relapse

T-cell prolymphocytic leukemia (T-PLL) constitutes a rare and aggressive mature T-cell leukemia, often associated with rapid progression and a poor prognosis (median overall survival $[O S]<2$ years). At diagnosis about $2 / 3$ of patients already present with exponentially rising lymphocytosis, clinically relevant bone marrow infiltration, splenomegaly, and lymphadenopathy. Most treatments containing chemotherapeutics such as alkylating agents or purine analogs are of limited efficacy. The best results are currently achieved with the monoclonal antiCD52 antibody alemtuzumab. As a single agent or in more toxic combinations with chemotherapy, it induces overall response rates of $75-92 \%$ with $48-81 \%$ complete remissions (CRs) [1]. However, these responses are shortlived (median $\approx 12$ months) and available second-line options are mostly inefficient. Recent advances in the molecular concept of T-PLL helped to identify new vulnerabilities (i.e., p53 reactivation by nutlin derivatives and inhibitors of protein deacetylation or BCL2 antagonization by $\mathrm{BH} 3$ mimetics); however, their clinical implementation still needs to be seen $[2,3]$.

Long-term disease control has been observed so far only in a minor subset of patients. The most promising results were achieved in those who underwent allogeneic hematopoietic stem cell transplantation (allo-HSCT), preferably as a consolidation in first CR (with a 6-week wash-out period after alemtuzumab). Several retrospective studies indicated that allo-HSCT can improve progression-free survival (PFS) and OS in T-PLL patients, e.g., $21-40 \%$ OS at 3 years [4-7]. However, with a median age at diagnosis of 66 years, only $40-60 \%$ of T-PLL patients are eligible for an allo-HSCT procedure. Furthermore, the prolonged survival, confined to a $10-20 \%$ subset of allo-grafted patients, is obtained at the cost of a high transplantation-related mortality of $31-41 \%$ within the first 3 years [4-7]. The main causes of this considerable nonrelapse mortality (NRM) are graft-versus-host disease and infections, which seem to be independent of the intensity of the applied conditioning regimens. Acute and chronic graft-versus-host disease is seen in about $50 \%$ of allo-transplanted T-PLL patients [4,7].

There is still a disappointing relapse incidence of 41$70 \%$ at 3 years after allo-HSCT in T-PLL, although these numbers also include cases transplanted in the salvage setting [4-6]. Several studies indicated that a total body irradiation (TBI) as part of the conditioning might positively affect the relapse-free survival $[4,5,8]$. Most of the relapses in these few retrospective series occurred shortly after transplantation (median time from transplantation to relapse $10-16.5$ months) $[4,5,9,10]$. A long period karger@karger.com

(C) 2020 S. Karger AG, Basel

www.karger.com/aha

Karger"
PD Dr. med. Marco Herling

Department I of Internal Medicine, University of Cologne Building 74, Weyertal 115c

DE-50931 Cologne (Germany)

marco.herling@uk-koeln.de 
from diagnosis to transplantation had a negative impact on event-free survival (time to progression or death from any cause) [4].

Importantly, there are recent data from an EBMT prospective observational study that evaluated allo-HSCT (predominantly as a primary consolidation after an alemtuzumab-containing induction) in a cohort of 37 T-PLL patients [8]. Here the 4-year PFS was $30 \%$ with a 4 -year OS of $42 \%$. In their better predefined cohort (age 18-65 years, HLA matched, etc.) than the retrospective series, the endpoints of median PFS (19.2 months) and median OS (27.8 months) appeared only slightly improved, while NRM seemed lower (32\% at 4 years). The 4 -year relapse incidence was $38 \%$ with $11 / 13$ (85\%) of the recurrences occurring within 24 months after transplantation. A TBI with $>6$ Gy was shown to reduce relapse incidence and an interval between diagnosis and transplantation of $>12$ months was associated with lower NRM [8].

Given these data, is there something new to learn from retrospective data on allo-HSCT in T-PLL? In this issue of Acta Haematologica, Shumilov et al. [11] report a retrospective single-center analysis of $10 \mathrm{~T}$-PLL patients who received an allo-HSCT between 2004 and 2019 after a median time from diagnosis to transplantation of 6 months. All 10 patients had an alemtuzumab-containing induction or re-induction regimen. Seven of them received alemtuzumab exclusively, while 1 CHOEP failure was salvaged by alemtuzumab and 2 alemtuzumab failures were brought to transplantation by polychemotherapy. Conditioning was fludarabine/cyclophosphamide based (notably no TBI). Seven patients entered the HSCT in CR. The 3 relapsed/refractory patients died within the first month after transplantation ( 2 in progressive disease (PD), 1 from infection).

The authors observed PD (5/10) or NRM (5/10) in all 10 patients and the 2-year OS of $30 \%$ and the 4-year OS of $20 \%$ was below the OS in the prospective EBMT series [8]. The high NRM in this series [11] was primarily caused by fatal infections, underlining the relevance of infectious complications in the post-transplantation management. CMV reactivations were observed in $6(60 \%)$ patients with a lethal infection in 1 case.

The value of this series is to raise awareness of the occurrence of late relapses after initial remissions post-alloHSCT. In 3 of their patients, all transplanted in CR and initially showing a complete donor chimerism, PD was observed at 12, 59, and 84 months after transplantation. These relapses were rapidly progressive and mostly extranodal. They displayed a pronounced resistance to salvage therapies (i.e., DLIs, bendamustine, pentostatin). Also, re-exposure to alemtuzumab could not prevent further progression despite expression of CD52.

Although most relapses develop within the first 2 years after allo-HSCT $[4,8]$ and stable long-lasting (>10 years) remissions are described in T-PLL [10], Shumilov et al. [11] illustrate that late progression is not uncommon [5, 11]. In these cases, persistence of T-PLL often remains undetected by conventional monitoring of donor chimerism. A PCR-based assessment of minimal residual disease (MRD) kinetics was reported by Sellner et al. [12] and confirmed the presence of graft-versus-leukemia effects in T-PLL. In line with the results by Shumilov et al. [11], they observed a significant reduction of MRD levels upon immunomodulation in $7 / 10$ patients, but with complete MRD clearance in only 2 patients.

Overall, although currently being the best available option in T-PLL, the systematic data on allo-HSCT indicate only marginal improvements of disease control and NRM over the past 2 decades when transplantation was performed as primary consolidation after alemtuzumab induction. Therefore, allo-HSCT and peri-transplant antileukemic therapies require profound optimizations. The depth of remission before entering transplantation is a main parameter that needs improvement. Further analyses also need to better identify patients who are likely to benefit from allo-HSCT versus those for whom alternative treatment approaches (e.g., targeted therapies) are more suitable. Other immuno-therapeutic options for TPLL are to be explored as well [2]. As for now, allo-HSCT continues to be a challenge, providing long-term disease control only for a small subset of eligible patients and being associated with considerable side effects.

\section{Disclosure Statement}

M.H. holds patents on TCL1 monoclonal antibodies (research and diagnostic use) with royalties paid.

\section{Funding Sources}

L.W. is supported by the Köln Fortune program of the University of Cologne. M.H. is supported by the DFG Research Unit FOR1961 (Control-T; HE3553/4-2). The European Union supports M.H. as part of the Transcan-II initiative (ERANETPLL) and as part of EraPerMed JAKSTATTARGET.

\section{Author Contributions}

The manuscript was conceived and written by L.W. and M.H. 


\section{References}

1 Hopfinger G, Busch R, Pflug N, Weit N, Westermann A, Fink AM, et al. Sequential chemoimmunotherapy of fludarabine, mitoxantrone, and cyclophosphamide induction followed by alemtuzumab consolidation is effective in T-cell prolymphocytic leukemia. Cancer. 2013 Jun;119(12):2258-67.

2 Braun T, von Jan J, Wahnschaffe L, Herling $\mathrm{M}$. Advances and perspectives in the treatment of T-PLL. Curr Hematol Malig Rep. 2020. DOI: 10.1007/s11899-020-00566-5.

3 Schrader A, Crispatzu G, Oberbeck S, Mayer $\mathrm{P}$, Pützer S, von Jan J, et al. Actionable perturbations of damage responses by TCL1/ATM and epigenetic lesions form the basis of TPLL. Nat Commun. 2018 Feb;9(1):697.

4 Wiktor-Jedrzejczak W, Dearden C, de Wreede L, van Biezen A, Brinch L, Leblond V, et al.; EBMT Chronic Leukemia Working Party. Hematopoietic stem cell transplantation in T-prolymphocytic leukemia: a retrospective study from the European Group for Blood and Marrow Transplantation and the Royal Marsden Consortium. Leukemia. 2012 May;26(5):972-6.
5 Guillaume T, Beguin Y, Tabrizi R, Nguyen S, Blaise D, Deconinck E, et al. Allogeneic hematopoietic stem cell transplantation for T-prolymphocytic leukemia: a report from the French society for stem cell transplantation (SFGM-TC). Eur J Haematol. 2015 Mar; 94(3):265-9.

6 Yamasaki S, Nitta H, Kondo E, Uchida N, Miyazaki $\mathrm{T}$, Ishiyama $\mathrm{K}$, et al. Effect of allogeneic hematopoietic cell transplantation for patients with T-prolymphocytic leukemia: a retrospective study from the Adult Lymphoma Working Group of the Japan Society for hematopoietic cell transplantation. Ann Hematol. 2019 Sep;98(9):2213-20.

7 Herling M. Are we improving the outcome for patients with T-cell prolymphocytic leukemia by allogeneic stem cell transplantation? Eur J Haematol. 2015 Mar;94(3):191-2.

8 Wiktor-Jedrzejczak W, Drozd-Sokolowska J, Eikema DJ, Hoek J, Potter M, Wulf G, et al. EBMT prospective observational study on allogeneic hematopoietic stem cell transplantation in T-prolymphocytic leukemia (T-PLL). Bone Marrow Transplant. 2019 Sep;54(9): 1391-8.
9 Krishnan B, Else M, Tjonnfjord GE, Cazin B, Carney D, Carter J, et al. Stem cell transplantation after alemtuzumab in T-cell prolymphocytic leukaemia results in longer survival than after alemtuzumab alone: a multicentre retrospective study. Br J Haematol. 2010 Jun; 149(6):907-10.

10 Dholaria BR, Ayala E, Sokol L, Nishihori T, Chavez JC, Hussaini M, et al. Allogeneic hematopoietic cell transplantation in T-cell prolymphocytic leukemia: a single-center experience. Leuk Res. 2018 Apr;67:1-5.

11 Shumilov E, Hasenkamp J, Szuszies CJ, Koch R, Wulf GG. Patterns of late relapse after allogeneic hematopoietic stem cell transplantation in patients with T-cell prolymphocytic leukemia. Acta Haematol. 2020. DOI: 10.1159/000506302.

12 Sellner L, Brüggemann M, Schlitt M, Knecht H, Herrmann D, Reigl T, et al. GvL effects in T-prolymphocytic leukemia: evidence from MRD kinetics and TCR repertoire analyses. Bone Marrow Transplant. 2017 Apr;52(4): $544-51$ 